\title{
Thermal and Crystallization Behavior of PLA/PLLA-Grafting Cellulose Nanocrystal
}

\author{
Erika M. Inácio, Diego H. S. Souza, Marcos L. Dias* \\ Instituto de Macromoléculas Professora Eloisa Mano (IMA), Universidade Federal do Rio de Janeiro (UFRJ), Rio de Janeiro, \\ Brazil \\ Email: ^mldias@ima.ufrj.br
}

How to cite this paper: Inácio, E.M., Souza, D.H.S. and Dias, M.L. (2020) Thermal and Crystallization Behavior of PLA/PLLAGrafting Cellulose Nanocrystal. Materials Sciences and Applications, 11, 44-57. https://doi.org/10.4236/msa.2020.111004

Received: December 3, 2019

Accepted: January 5, 2020

Published: January 8, 2020

Copyright (c) 2020 by author(s) and Scientific Research Publishing Inc. This work is licensed under the Creative Commons Attribution International License (CC BY 4.0).

http://creativecommons.org/licenses/by/4.0/

(c) (i) Open Access

\begin{abstract}
PLLA-modified cellulose nanocrystals (CNC) were produced from commercial CNC by tin-catalyzed polymerization of lactide in presence of CNC. FTIR spectroscopy demonstrated that the result of the reaction produced the grafting of PLLA chains onto CNC surface (CNC-g-PLLA). Films of poly(lactic acid) (PLA) and PLA/CNC nanocomposites (with non-modified CNC and CNC-g-PLLA) containing $0.5 \%$ and $5 \%(\mathrm{w} / \mathrm{w})$ of the nanofillers were prepared by casting in chloroform solution and the crystallization behavior and thermal properties investigated. All nanocomposites had similar thermal stability when analyzed by TGA analyses under an inert nitrogen atmosphere. Addition of both types of CNC influenced crystallization, the higher crystallization rate being observed for $5 \%(\mathrm{w} / \mathrm{w})$ CNC. Nanocomposites with $5 \%$ $(\mathrm{w} / \mathrm{w})$ CNC-g-PLLA had the strain resistance of PLA improved in the rubbery state. PLLA-modification of CNC surface increased the crystallization of PLA in PLA/CNC nanocomposites and improved the rigidity at temperatures above the glass transition, properties which are desirable for hot drinking application.
\end{abstract}

\section{Keywords}

Nanocrystals, Cellulose, Poly(Lactic Acid), Grafting, Crystallizations

\section{Introduction}

Accumulation of plastic objects and particles as plastic bottles, bags and microbeads in the Earth's environment has adversely affected wildlife habitat and humans. Thus, in recent years, there has been a growing interest in the development of environmentally friendly materials, as well as a need to replace synthetic polymers by biodegradable polymer materials, such as poly(lactic acid) (PLA), 
especially in packaging [1] [2]. Cellulose-based materials are interesting biodegradable fillers and have shown to be able to improve the properties of PLA by affecting the crystallinity, and mechanical and thermal properties [3] [4]. In this sense, in films for packaging applications, nanosized cellulose like celullose nanocrystals (CNC) can be an interesting option since improvement of PLA mechanical properties can be reached at low filler content [5] [6] [7] [8]. Nevertheless, mixing of CNC and PLA faces many obstacles related to interfacial incompatibility due to hydrophilic nature of CNC surface and hydrophobic nature of PLA chains. A good way to make compatible CNC with PLA is to graft PLA chains on CNC surface, considering that this CNC surface modification can, in principle, increase the interaction between the filler and the matrix. In addition, such a kind of composite can potentially result in 100\% compostable materials [9].

In this work, we report the crystallization and thermal behavior of PLLA-grafting CNC synthesized by in-situ polymerization of L-lactide with the presence of $\mathrm{CNC}$ as well as PLA/CNC nanocomposites films prepared by Solution Casting Technique. The effect of reaction conditions on these properties of CNC-PLLA nanomaterials is also reported.

\section{Experimental}

\subsection{Materials}

Film grade PLA Ingeo 2003D $\left(\mathrm{M}_{\mathrm{w}}=185.000 \mathrm{~g} \cdot \mathrm{mol}^{-1}\right)$ from NatureWorks was used as received. Commercial cellulose nanocrystals were furnished by CelluForce (FribriaCellulose, Brazil). L-lactide (Purasorb L) was supplied by Corbion, Netherland. Tin II octanoate (Aldrich, USA) was used as received and toluene and chloroform were purified by distillation.

\subsection{Cellulose Nanocrystal Surface Modification with Lactide (CNC-g-PLLA)}

Commercial CelluForce CNC was vacuum dried at $80^{\circ} \mathrm{C}$ for $12 \mathrm{~h}$. CNC-g-PLLA nanoparticles were prepared by in-situ ring-opening polymerization of L-lactide. Initially, lactide was introduced into a round bottom glass flask and heated at $100^{\circ} \mathrm{C}$ up to the complete melting. CNC and a $15 \%$ (v/v) solution of tin II octanoate in chloroform were added to the flask which was magnetically stirred. The temperature was then raised to $110^{\circ} \mathrm{C}$ and the reaction system maintained at this temperature for $2 \mathrm{~h} 30 \mathrm{~min}$ under nitrogen atmosphere. At the end of reaction, the material was solubilized in chloroform, and precipitated in ethanol, resulting in a solid containing probably PLLA grafted onto CNC particles (CNC-g-PLLA) and PLLA. The solid product was dissolved in chloroform and centrifugated to separate CNC-g-PLLA from free PLLA. This procedure was repeated 3 times. CNC-g-PLLA was collected after a final centrifugation.

\subsection{Preparation of PLA and PLA/CNC Films}

Films were prepared by casting PLA and PLA/CNC chloroform solutions (10\% 
w/v) over covered glass plates. Chloroform was added to commercial CNC and CNC-g-PLLA and the mixture maintained in an ultrasonic bath for $1.5 \mathrm{~h}$ until a homogeneous dispersion been obtained. PLA chloroform solution was then added to these dispersions and the dispersions stirred for $24 \mathrm{~h}$ up to the complete solubilization of the polymer. Films were obtained after drying the casting solutions for 7 days at room temperature. Films of neat PLA, and PLA/CNCs containing $0.5 \%$ and $5.0 \%(\mathrm{w} / \mathrm{w})$ of fillers were prepared.

\subsection{Cellulose Nanocrystals Characterization}

CNC and CNC-g-PLLA morphology were examined by Transmission Electron Microscopy (Fei Tecnai Spirit) with accelerating voltage $120 \mathrm{kV}$ and tungsten wire cannon. Dilute suspension of CNC was dropped on an ultra-thin copper substrate (coated with a carbon thin film, 400 mesh) and dry at room temperature. Fourier Transform Infrared Spectroscopy (FT-IR) was used to investigate the chemical modification in a Varian Excalibur 3100 equipment. Thermal stability of the analyzed samples was evaluated using TGA analyzer (TA instruments Q500) from room temperature to $700^{\circ} \mathrm{C}$ at a heating rate of $10^{\circ} \mathrm{C} / \mathrm{min}$ under a nitrogen atmosphere. X-ray diffraction was used to determine the crystallinity on an X-ray diffractometer (X'Pert Pro, PANalytical, Netherlands) at room temperature with a monochromatic $\mathrm{CuK} \alpha$ radiation source $(\lambda=0.154 \mathrm{~nm})$ in the step-scan mode with a $2 \theta$ angle ranging from $4^{\circ}$ to $50^{\circ}$ with a step of 0.04 and scanning time of $5.0 \mathrm{~min}$.

\subsection{Nanocomposite Films Characterization}

Thermogravimetric analyses were performed on a TGA Q500 TA Instruments under inert atmosphere (nitrogen flow, $10 \mathrm{~mL} / \mathrm{min}$ ) from room temperature to $600^{\circ} \mathrm{C}$. Crystallinity and melting behavior of the films were evaluated using a DSC Q-1000 (TA Instruments). Samples were first heated from $20^{\circ} \mathrm{C}$ to $200^{\circ} \mathrm{C}$ at $20^{\circ} \mathrm{C} / \mathrm{min}$ and held for $3 \mathrm{~min}$ to erase the thermal history. Subsequently, it was cooled at $50^{\circ} \mathrm{C} / \mathrm{min}$ to the prescribed crystallization temperature $\left(100^{\circ} \mathrm{C}\right)$ and held for enough time to fully crystallize. Samples were then, cooled at $20^{\circ} \mathrm{C} / \mathrm{min}$ to $20^{\circ} \mathrm{C}$ and held for $3 \mathrm{~min}$. Finally, the sample was heated again to $220^{\circ} \mathrm{C}$ at $10^{\circ} \mathrm{C} / \mathrm{min}$ to obtain the melting temperature $\left(T_{\mathrm{m}}\right)$. The analyses were carried out under nitrogen atmosphere using a flow rate of $20 \mathrm{~mL} / \mathrm{min}$.

\section{Results and Discussion}

\subsection{Nanocrystals Morphology, Structure and Thermal Features}

Figure 1 shows the representative TEM image of commercial CNC. As expected, CNC showed a rod-like shape reported in the literature [10]. CNC showed a mean length of $107 \pm 36 \mathrm{~nm}$.

In-situ polymerization of L-lactide on hydroxylated CNC surface may produce PLLA chains covalently bonded to CNC (CNC-g-PLLA) (Figure 2).

Figure 3 shows the FTIR spectra of unmodified CNC and PLLA-modified 


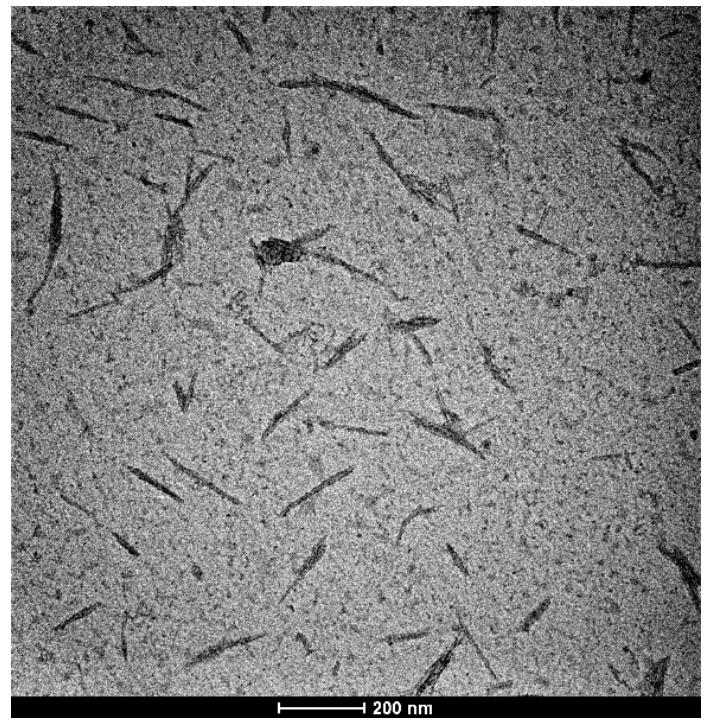

Figure 1. TEM image of CelluForce commercial CNC.

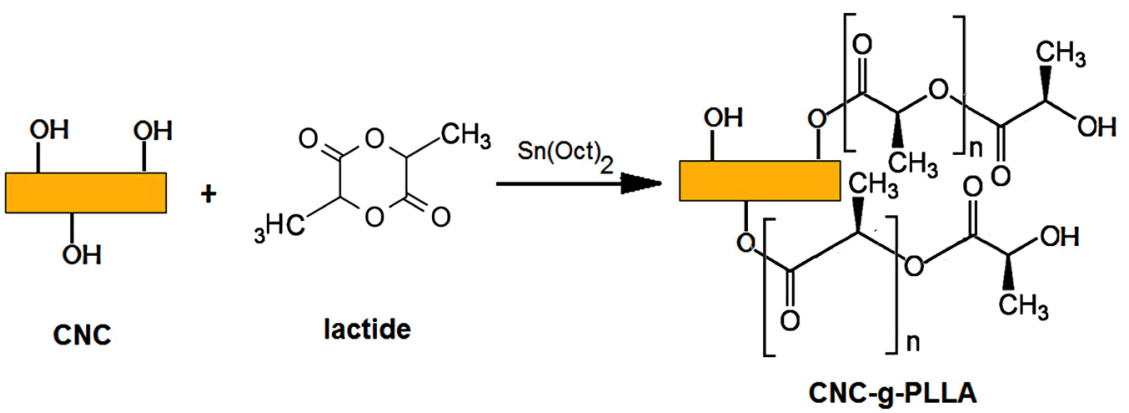

Figure 2. Grafting reaction of L-lactide over CNC surface.

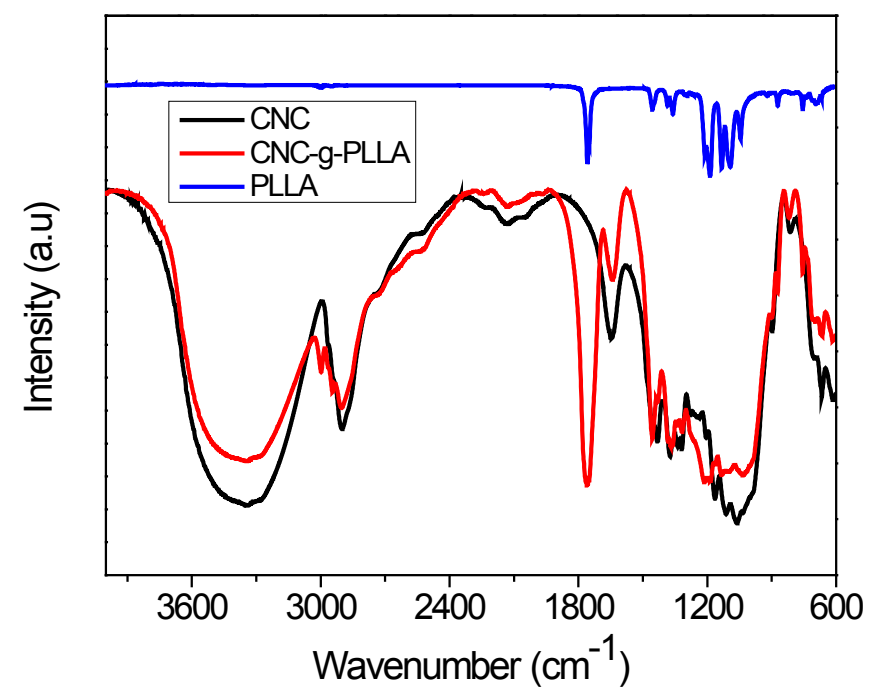

Figure 3. FTIR spectra of CNC and CNC-g-PLLA.

CNC. The spectrum of CNC presents a characteristic broad absorption band at $3400 \mathrm{~cm}^{-1}$ which corresponds to $\mathrm{O}-\mathrm{H}$ groups stretching vibration of cellulose. In 
addition, others feature bands appear at $2900 \mathrm{~cm}^{-1}$ (asymmetric and symmetric stretching of methyl and methylene $\mathrm{C}-\mathrm{H}$ groups), at $1640 \mathrm{~cm}^{-1}$ (bending vibration $\mathrm{O}-\mathrm{H}$ groups), at $1430 \mathrm{~cm}^{-1}\left(\mathrm{CH}_{2}\right.$ bending), at $1160 \mathrm{~cm}^{-1}$ (C-O-C asymmetric bending) and $898 \mathrm{~cm}^{-1}$ (C-O-C asymmetric bending). Grafting of PLLA chains onto CNC surfaces was confirmed by the presence of a band at $1746 \mathrm{~cm}^{-1}$ that corresponds to the $\mathrm{C}=\mathrm{O}$ ester stretching vibration in CNC-g-PLLA [11] [12].

Figure 4 shows the X-ray diffraction (XRD) curves of PLA pellet, commercial CNC and CNC-g-PLLA. PLA pellet exhibited clear diffraction peaks located at $2 \theta=15.0,16.7,19.1,22.4,27.4^{\circ}$ corresponding to the (010), (110/200), (203), (204), (015), and (207) planes, respectively [13]. Lower intensity peaks located were also observed at $2 \theta=29.1$ e $31.2^{\circ}$.

XRD curves of CNC presented a broad reflection at $2 \theta=14^{\circ}-17^{\circ}$ and a sharp peak at $2 \theta=22.5$ and $34.5^{\circ}$ which is corresponding to (110), (200) and (040) planes of cellulose I crystalline structure, respectively [11]. CNC-g-PLLA showed the PLLA crystalline peaks, which confirms the presence of PLLA chains bonded onto the surface of the nanocrystals. Degree of crystallinity of $68 \%$ and $70 \%$ for $\mathrm{CNC}$ and CNC-g-PLLA was found, respectively. These values are in accordance with expected values for CNC reported in the literature [14], which consider small variations in the degree of crystallinity to variation of acid concentration used to produce nanocrystals from cellulose hydrolysis. For CNC-g-PLLA, presence of crystalline peaks of PLLA seems to cause the apparent decrease in the intensity of $\mathrm{CNC}$ crystalline peaks due to the decrease of CNC content in the modified filler. According to Cetin et al. [15], during the progress of modification reaction, internal crystalline regions can be modified.

TG curves of PLA, CNC and CNC-g-PLLA are shown in Figure 5. TG curve of CNC presented an initial weight loss of $6 \%$ which was attributed to water evaporation, while for CNC-g-PLLA this weight loss was $50 \%$ lower. Considering

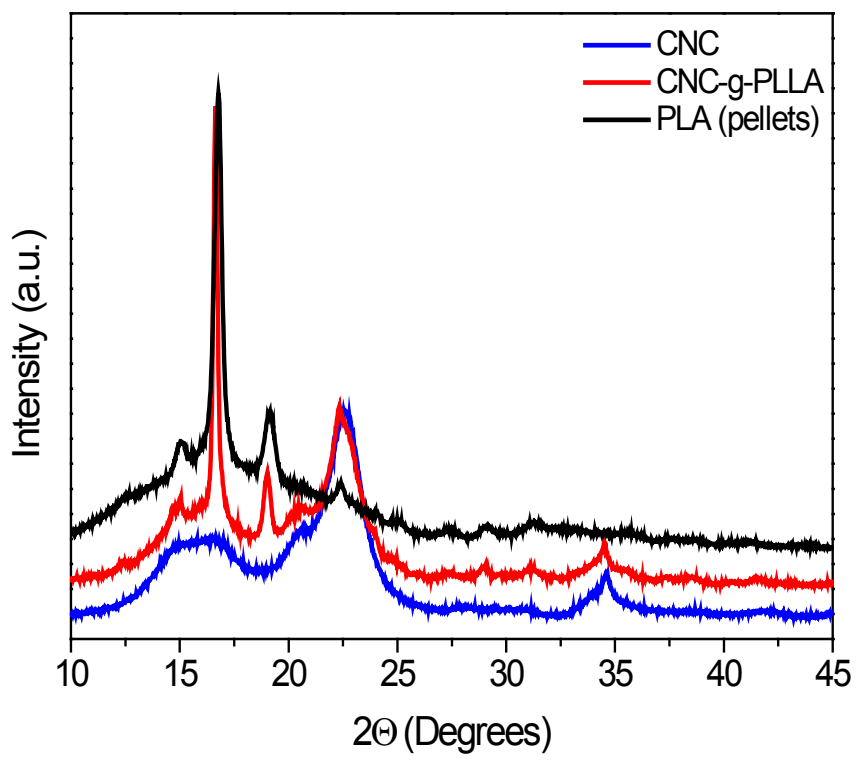

Figure 4. XRD curves of PLA (pellets), CNC and CNC-g-PLLA. 


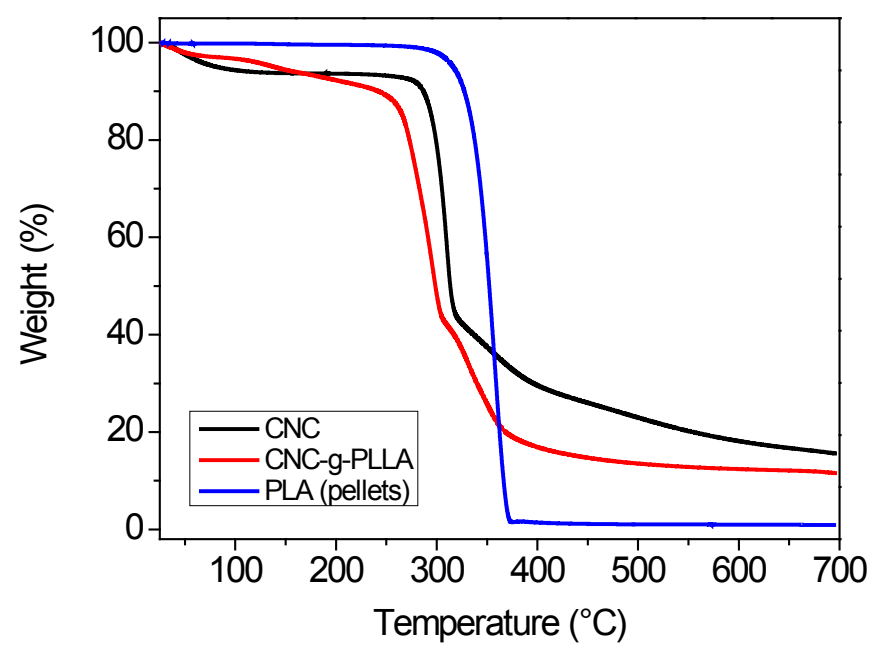

Figure 5. TG curves of PLA, CNC and CNC-g-PLLA.

that both samples were dried under the same conditions, the percent moisture reduction can be associated with the decrease of hydroxyl groups due to grafting of PLLA chains onto CNC surface. A difference in the values of residue was found for the $\mathrm{CNC}$ and CNC-g-PLLA. CNC presented $16 \%$ of residue at $700^{\circ} \mathrm{C}$, while for CNC-g-PLLA the residue was $12 \%$. The difference was attributed to the presence of PLLA chains on the nanoparticles.

According to the supplier, the commercial CNC used in this work was obtained by sulfuric acid hydrolysis. This information could be confirmed by the DTG curve of the CNC, which showed three stages of weight loss, the first around $50^{\circ} \mathrm{C}$ probably due to evaporation of absorbed water. The others two are related to the cellulose structure, with sulfate moieties accelerating the weight loss of glycoside components [16].

It is important to emphasize that although the weight loss profile of CNC and CNC-g-PLLA are similar, CNC showed higher thermal stability since the onset temperature is up to $100^{\circ} \mathrm{C}$ higher.

For CNC-g-PLLA it is possible to observe two well defined stages of weight loss, in addition to the water loss. The first one is related to the glycoside components and the second one manifested as a shoulder seems to be related to sulfate groups still present in the structure, overlapped with weight loss attributed to lactide, since it occurs at the same temperature range for PLLA [16] [17].

\subsection{Thermal Transitions of PLA/CNC Films}

Films of PLA and PLA/CNC nanocomposites (PLA/CNC and PLA/CNC-g-PLLA) were prepared by casting and the thermal behavior investigated by DSC. Figure 6 presents the DSC traces obtained from the first heating of these films, while Figure 7 shows the DSC traces of a second heating run, after a first heating and subsequent quenching of the samples. Table 1 resumes the data of main transitions, melting enthalpy and degree of crystallinity $\left(X_{c}\right)$ of PLA and PLA/CNC nanocomposites. 


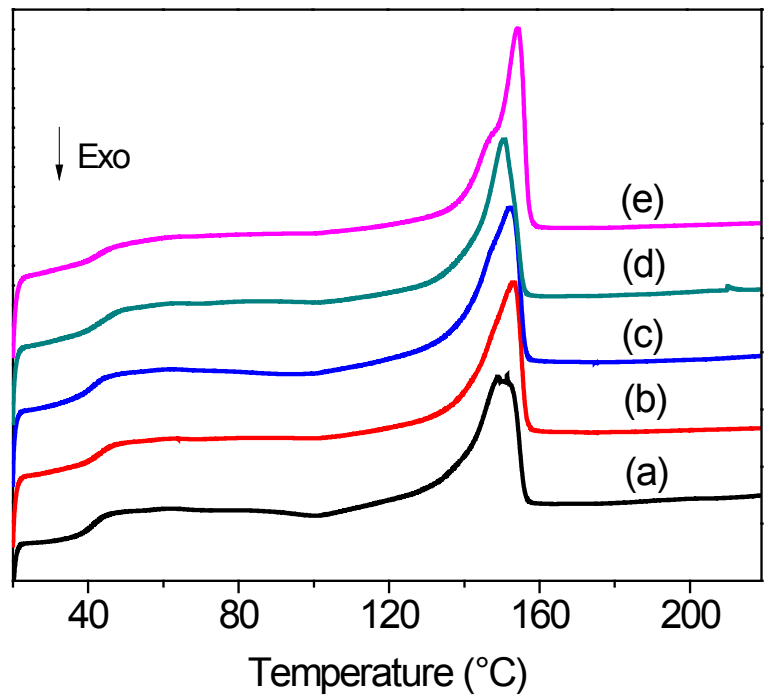

Figure 6. DSC curves ( $1^{\text {st }}$ heating run) of (a) PLA and PLA nanocomposites containing (b) $0.5 \%$ CNC, (c) $0.5 \%$ CNC-g-PLLA, (d) 5.0\% CNC-g-PLLA, and (e) 5.0\% CNC.

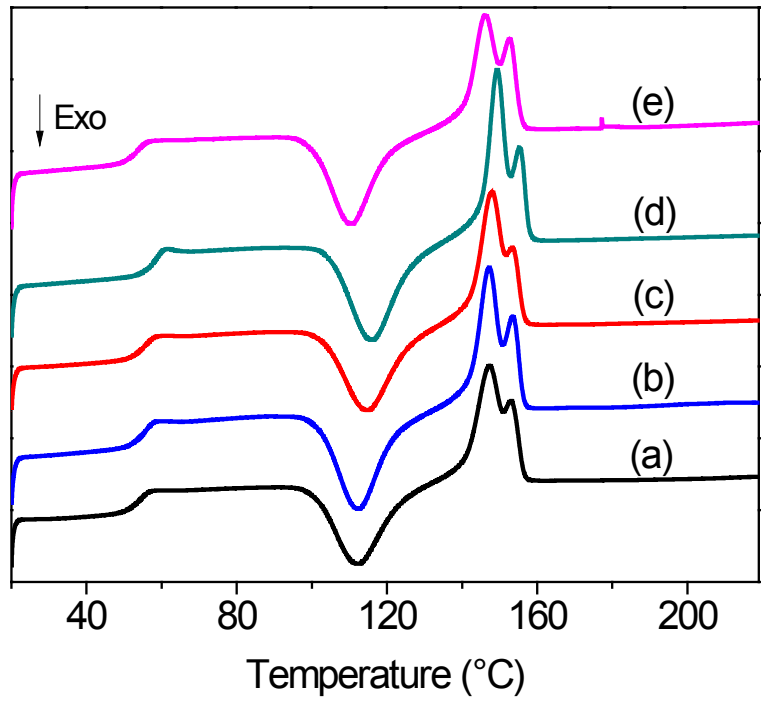

Figure 7. DSC curves ( $2^{\text {nd }}$ heating run) of (a) PLA and PLA nanocomposites containing (b) $0.5 \%$ CNC, (c) $0.5 \%$ CNC-g-PLLA, (d) $5.0 \%$ CNC-g-PLLA, and (e) $5.0 \%$ CNC.

Table 1. Thermal transitions, melting enthalpy and degree of crystallinity of PLA, PLA/CNC and PLA/CNC-g-PLLA films obtained by casting ${ }^{a}$.

\begin{tabular}{cccccccccc}
\hline Sample & $\begin{array}{c}T_{\mathrm{g}} \\
\left({ }^{\circ} \mathrm{C}\right)\end{array}$ & $\begin{array}{c}T_{\mathrm{m}} \\
\left({ }^{\circ} \mathrm{C}\right)\end{array}$ & $\begin{array}{c}T_{\mathrm{m}} 1^{\mathrm{d}} \\
\left({ }^{\circ} \mathrm{C}\right)\end{array}$ & $\begin{array}{c}T_{\mathrm{m}} 2^{\mathrm{d}} \\
\left({ }^{\circ} \mathrm{C}\right)\end{array}$ & $\begin{array}{c}T_{\mathrm{cc}} \\
\left({ }^{\circ} \mathrm{C}\right)\end{array}$ & $\begin{array}{c}\Delta H_{\mathrm{cc}} \\
(\mathrm{J} / \mathrm{g})\end{array}$ & $\begin{array}{c}\Delta H_{\mathrm{m}} \\
(\mathrm{J} / \mathrm{g})\end{array}$ & $\begin{array}{c}X_{\mathrm{c}}^{\mathrm{a}} \\
(\%)\end{array}$ & $\begin{array}{c}X_{\mathrm{c}}^{\mathrm{b}} \\
(\%)\end{array}$ \\
\hline PLA & 55 & 151 & 147 & 153 & 113 & 24 & 31 & 29 & 14 \\
PLA/CNC 0.5\% & 56 & 153 & 148 & 153 & 115 & 24 & 31 & 30 & 29 \\
PLA/CNC 5.0\% & 54 & 150 & 146 & 153 & 111 & 24 & 28 & 28 & 31 \\
PLA/CNC-g-PLLA 0.5\% & 56 & 152 & 147 & 153 & 112 & 25 & 35 & 34 & 29 \\
PLA/CNC-g-PLLA 5.0\% & 59 & 154 & 149 & 155 & 116 & 27 & 32 & 32 & 33 \\
\hline
\end{tabular}

a obtained by DSC; bobtained by XRD; c,dobtained of the first and second heating, respectively. 
The first DSC heating run of all films (Figure 1) showed a glass transition and a melting peak, indicating the semi-crystalline nature of the materials. No cold crystallization was observed, suggesting that these materials present the high $X_{\mathrm{c}}$ which is commonly found for PLA casting films. In fact, the results of Table 1 show that addition of both unmodified and modified-CNC increased $X_{c}$, the fact noted using either DSC or XRD. The melting peaks were monomodal $\left(T_{\mathrm{m}}\right.$, $150^{\circ} \mathrm{C}-154^{\circ} \mathrm{C}$ ), excepting for the nanocomposite with the higher content of unmodified $\mathrm{CNC}(5 \% \mathrm{w} / \mathrm{w})$, where a shoulder appears at lower temperature.

Aiming to see the influence of CNC on PLA thermal transitions without the influence of cast film formation, DSC traces of a $2^{\text {nd }}$ heating runs after melting and fast cooling of the films were carried out in PLA and PLA/CNC nanocomposites (Figure 7). All the curves show 3 distinct transitions: the glass transition, the cold crystallization and the melting transition. Practically, no difference in $T_{\mathrm{g}}$ was noted by the addition of the nanofillers, except for the nanocomposite with $5.0 \%(\mathrm{w} / \mathrm{w}) \mathrm{CNC}$-g-PLLA, where $T_{\mathrm{g}}$ increase about $4^{\circ} \mathrm{C}$. Also, this composite showed the highest cold crystallization temperature, $T_{\mathrm{cc}}\left(T_{\mathrm{cc}}=116^{\circ} \mathrm{C}\right)$. This increase of $T_{\mathrm{cc}}$ suggests that this higher content of the PLLA-modified CNC is hindering crystallization during the heating. It is important to mention that no crystallization was observed by cooling either the PLA used in this work or the PLA/CNC nanocomposites.

A bimodal melting peak was noted for all samples. The two peaks appear in two temperature ranges: $T_{\mathrm{m}} 1=146^{\circ} \mathrm{C}-149^{\circ} \mathrm{C}$ and $T_{\mathrm{m}} 2=153^{\circ} \mathrm{C}-155^{\circ} \mathrm{C}$ and are related to two different families of crystals which may differentiate by crystal perfection. It is important to note that the increase of nanofiller content increases the amount of $T_{\mathrm{m}} 2$ crystals.

\subsection{Thermal Stability of PLA/CNC Films}

Figure 8 presents the DTG curves of films of PLA and PLA/CNC nanocomposites where information on the thermal stability of the films could be derived. Addition of both modified and non-modified CNC to PLA did not cause any significant change in the temperature of maximum degradation rate $\left(T_{\max }\right)$. The same profile is observed also as a function of filler concentration, independent on the CNC surface modification. It means that all PLA and nanocomposites had similar thermal stability. This result is in agreement with what was previous reported [18], where no difference in thermal stability was noted for nanocomposites of PLA and lactide- and acetic acid-modified CNCs when TGA analyses were performed under an inert nitrogen atmosphere. It must be emphasized the presence of a peak around $300^{\circ} \mathrm{C}$ for the samples with $5 \%(w / w)$ filler, which seems to be related to the filler degradation present in higher amount.

The crystallinity of the nanocomposites was evaluated by X-ray diffraction. According to Figure 9, only the characteristic peaks of PLA can be observed for the nanocomposites $\left(2 \theta=15,16.5,19\right.$ e $\left.22.5^{\circ}\right)$. An increase in the intensity of the plane at $2 \theta=16.5^{\circ}$ was seen when the filler concentration increases. This is an 


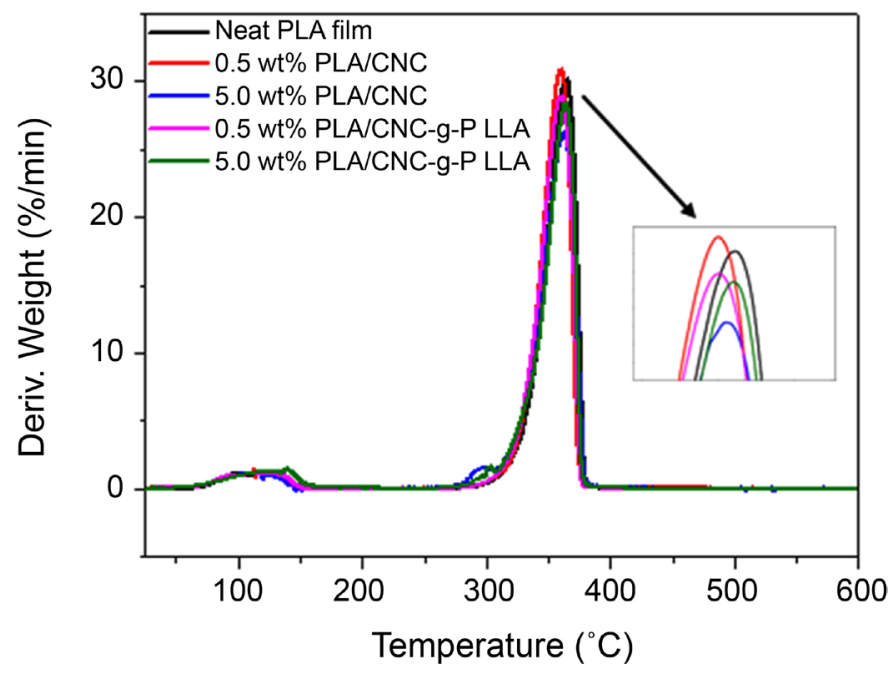

Figure 8. DTG curves of (a) PLA and PLA nanocomposites containing (b) $0.5 \%$ CNC, (c) $0.5 \%$ CNC-g-PLLA, (d) 5.0\% CNC-g-PLLA, and (e) 5.0\% CNC.

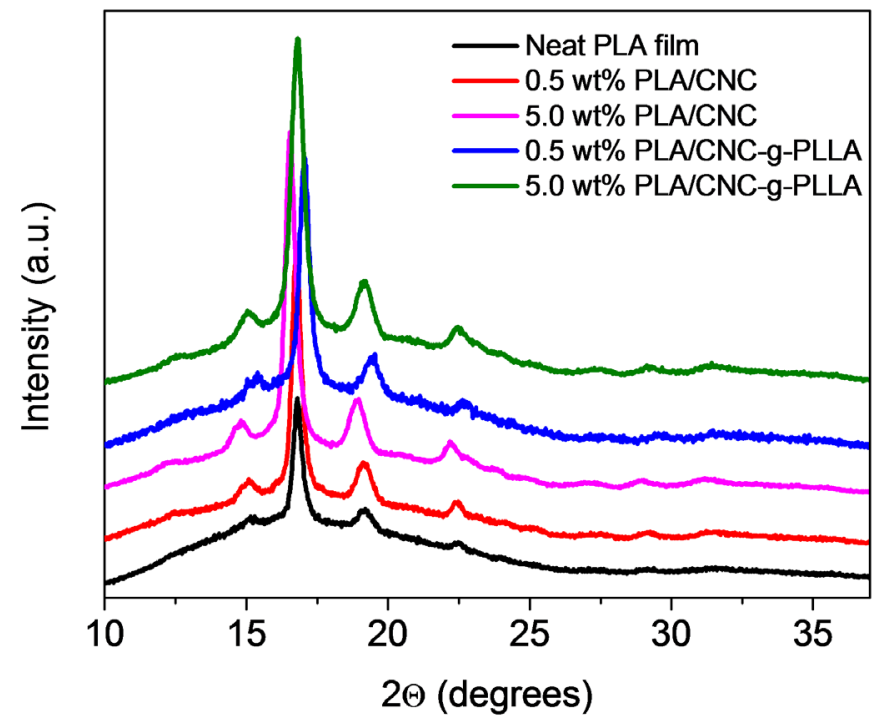

Figure 9. XRD curves of (a) PLA and PLA nanocomposites containing (b) $0.5 \%$ CNC, (c) 0.5\% CNC-g-PLLA, (d) 5.0\% CNC-g-PLLA, and (e) 5.0\% CNC.

evidence of the nucleation effect of both non-modified and PLLA-modified CNC.

\subsection{Crystallization Behavior of PLA/CNC Films}

The crystallization kinetics of the films was investigated and results of relative crystallinity versus crystallization time are presented in Figure 10.

From the figure, it can be clearly seen that, in general, addition of both nanofiller, decreased the crystallization time of PLA. However, it was not possible to observe a linear influence with the filler content and type. The nanocomposite with $0.5 \%(\mathrm{w} / \mathrm{w})$ of PLLA-modified CNC had similar crystallization kinetic of PLA and nanocomposite films with 5\% (w/w) of both CNC and CNC-g-PLLA 


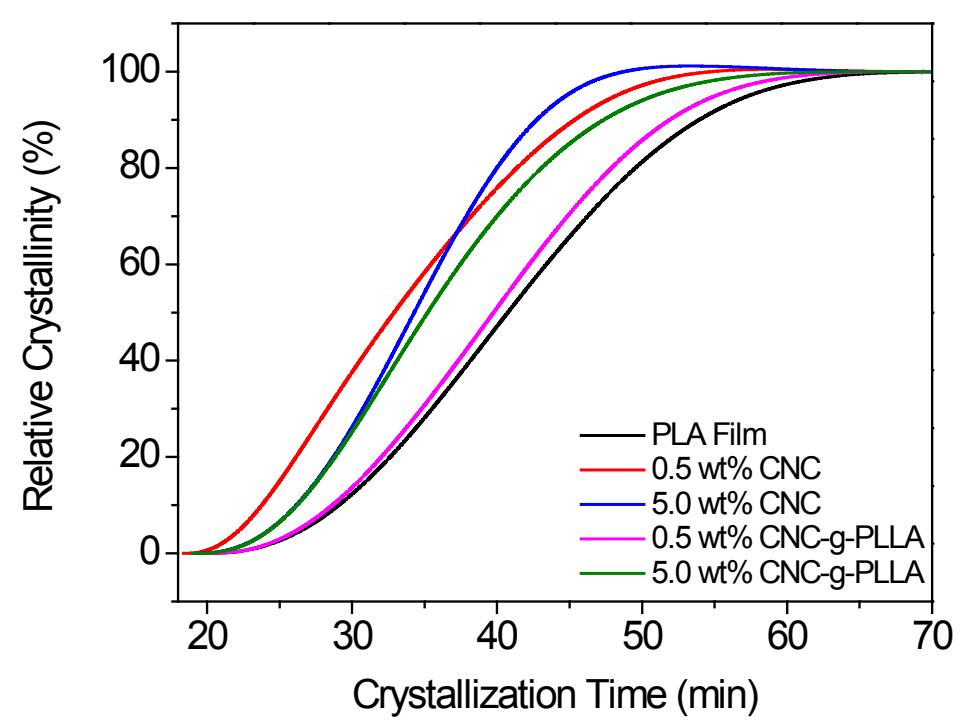

Figure 10. Relative crystallinity versus crystallization time of (a) PLA and PLA nanocomposites containing (b) $0.5 \%$ CNC, (c) $0.5 \%$ CNC-g-PLLA, (d) 5.0\% CNC-g-PLLA, and (e) $5.0 \%$ CNC.

showed the higher crystallization rate. The highest crystallization rate was attained for the nanocomposite containing $0.5 \%(\mathrm{w} / \mathrm{w})$ of unmodified CNC.

\subsection{Dynamic Mechanical Behavior of PLA/CNC Films}

The effect of CNCs on the mechanical behavior of PLA was investigated by DMA. The behavior of the storage modulus (E') as function of temperature is shown in Figure 11 for films of PLA and PLA/CNC nanocomposites containing $0.5 \%$ and $5 \%(\mathrm{w} / \mathrm{w})$ of non-modified and PLLA-modified CNC. All films showed the typical semi-crystalline behavior with high modulus below the glass transition, followed by an abrupt decrease in E' after the glass transition temperature $\left(T_{\mathrm{g}}\right)$, i.e., in the rubbery state. Table 2 presents values of $\mathrm{E}^{\prime}$ in the glassy and rubbery states at $30^{\circ} \mathrm{C}$ and $75^{\circ} \mathrm{C}$, respectively.

In comparison with unfilled PLA, increase of $\mathrm{E}^{\prime}$ was observed only for PLA/CNC nanocomposites containing the unmodified CNC. For example, at $30^{\circ} \mathrm{C}$, E' for PLA was $2228 \mathrm{MPa}$ and decreased to $1649 \mathrm{MPa}$ for the nanocomposite with $0.5 \%$ CNC-g-PLLA but increased to $2439 \mathrm{MPa}$ for the one with $0.5 \%$ unmodified CNC. Nevertheless, while small decrease of E' was noted at $30^{\circ} \mathrm{C}$ when the content of non-modified CNC was increased from $0.5 \%$ to $5 \%(2439$ and $2343 \mathrm{MPa}$, respectively), a significant increase of E' was observed for PLA nanocomposites containing CNC-g-PLLA (1649 and $2016 \mathrm{MPa}$, respectively). It seems that the presence of PLLA chains bonded on the CNC surface is producing PLA chains slippage, decreasing E'.

For the rubbery region $\left(75^{\circ} \mathrm{C}\right)$, a decrease in E' was observed for both composites containing $0.5 \%(\mathrm{w} / \mathrm{w})$, with a higher decrease also observed for composites with $0.5 \%$ CNC-g-PLLA (68 MPa). Increase of E' was just noted when $5 \%(w / w)$ of the nanofillers was used (PLA, $101 \mathrm{MPa}$; PLA/CNC and PLA/PLA/CNC-g-PLLA, 


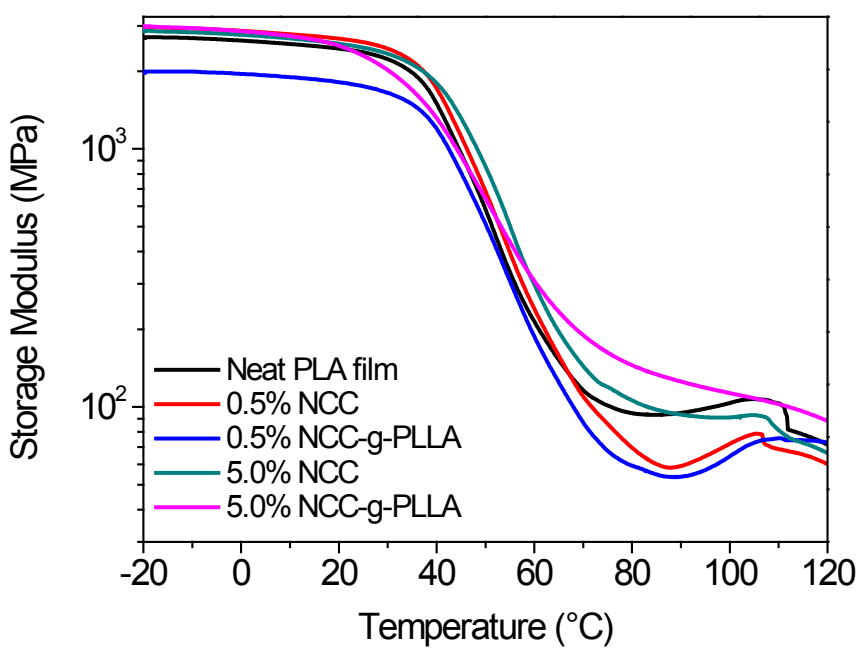

Figure 11. Storage modulus of (a) PLA and PLA nanocomposites containing (b) $0.5 \%$ CNC, (c) 0.5\% CNC-g-PLLA, (d) 5.0\% CNC-g-PLLA, and (e) 5.0\% CNC.

Table 2. Storage modulus in films of PLA, and PLA/CNC nanocomposites.

\begin{tabular}{ccc}
\hline Sample & \multicolumn{2}{c}{ Storage Modulus (MPa) } \\
\hline PLA & At $30^{\circ} \mathrm{C}$ & At $75^{\circ} \mathrm{C}$ \\
\hline $0.5 \%$ CNC & 2228 & 101 \\
$0.5 \%$ CNC-g-PLLA & 2439 & 87 \\
$5.0 \%$ CNC & 1649 & 68 \\
$5.0 \%$ CNC-g-PLLA & 2343 & 120 \\
\hline
\end{tabular}

120 and $163 \mathrm{MPa}$, respectively). For this nanofiller content, the higher value of E' (163 MPa) was attained for PLA/CNC-g-PLLA nanocomposites. It means that addition of CNC-g-PLLA improves the strain resistance of PLA in the rubbery state, i.e., above glass transition $\left(60^{\circ} \mathrm{C}\right)$.

It is also important to mention that after $T_{\mathrm{g}}$, the majority of the samples showed a progressive increase in E' with increasing temperature which indicated the cold crystallization phenomenon (Figure 11). Only the nanocomposite containing 5\% (w/w) CNC-g-PLLA did not show this behavior, demonstrating that this nanocomposite film had a high degree of crystallinity.

Spinella et al. [18] used the Tan d peak profile to verify the effect of surface top chemistry on the interaction between CNCs and PLA. According to the authors, since Tan $\delta$ is related to polymer chain relaxation, when the interaction between the filler and matrix increases, it is expected that the Tan $\delta$ peak is shifted to higher temperatures, the peak intensity decreases, and the transition is broadened. Results of Tan $\delta$ versus temperature for PLA and the PLA/CNC nanocomposites investigated in this work are presented in Figure 12. While no decrease in the peak intensity was noted for the composites with $0.5 \%$ and $5 \%$ (w/w) CNC, an intensity decrease was observed for both composites containing 


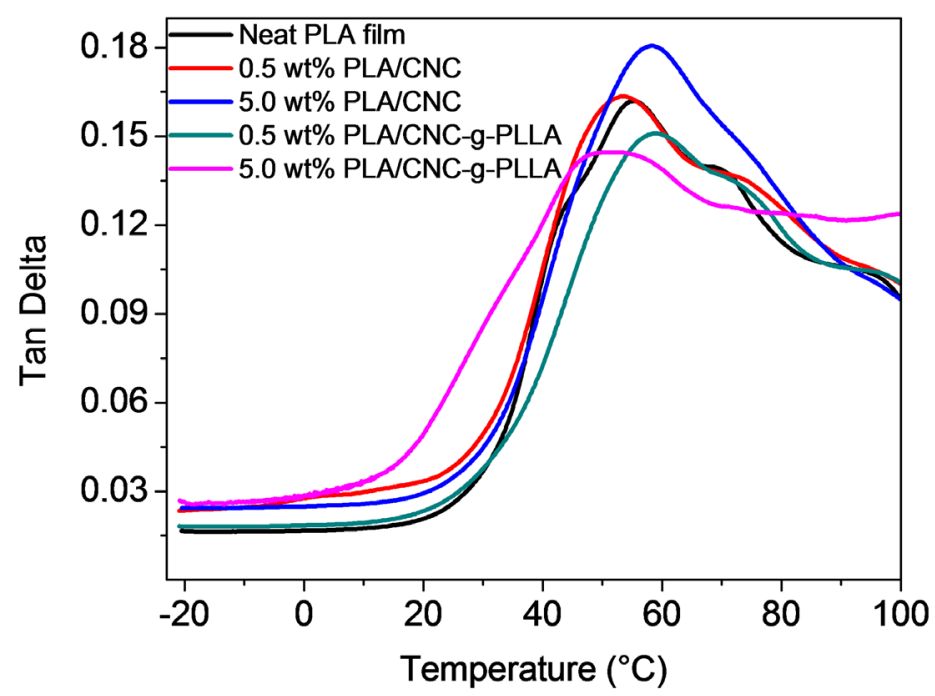

Figure 12. Tan $\delta$ of (a) PLA and PLA nanocomposites containing (b) $0.5 \%$ CNC, (c) 0.5 wt\% CNC-g-PLLA, (d) 5.0\% CNC-g-PLLA, and (e) 5.0\% CNC.

the PLLA-modified CNC. In addition, it is clearly seen that Tan $\delta$ peak is broad for the composite containing $5 \%(\mathrm{w} / \mathrm{w})$ CNC-g-PLLA, which suggests the better adhesion of the filler with the PLA matrix.

\section{Conclusion}

In this work, modification of commercial cellulose nanocrystals was performed by tin-catalyzed polymerization of lactide in presence of $\mathrm{CNC}$, producing $\mathrm{CNC}$ g-PLLA which was used to prepare PLA/CNC nanocomposite films. Presence of esters linkages by FTIR spectroscopy demonstrated that modification effectively occurs at nanocrystal surfaces. The crystallization behavior and thermal properties of PLA/CNC-g-PLLA films containing $0.5 \%$ and $5 \%(\mathrm{w} / \mathrm{w})$ of the nanofiller were compared with unfilled PLA and PLA/unmodified CNC films with the same filler content. From TGA analyses performed under an inert nitrogen atmosphere, all nanocomposites had similar thermal stability, regardless of CNC surface modification. Presence of both types of CNC decreased the crystallization time of PLA, with the higher crystallization rate observed for $5 \%(\mathrm{w} / \mathrm{w})$ CNC. Addition CNC-g-PLLA improves the strain resistance of PLA in the rubbery state, i.e., above glass transition $\left(60^{\circ} \mathrm{C}\right)$ more than in nanocomposites containing the unmodified CNC. The results herein conclusively show that PLLAmodification of CNCs surface can increase the crystallization of PLA in PLA/ CNC nanocomposites, improving also the rigidity of the material at temperatures above the glass transition, which may make possible application of this material in hot drinking.

\section{Acknowledgements}

Authors are grateful to Conselho Nacional de PesquisaCientífica e Tecnológica (CNPq) (Grants 310917/2014-0 and 307364/2018-6), FAPERJ (Grants E-26/ 
201.304/2014 and E-26/202.538/2019) and CAPES for financial support.

\section{Conflicts of Interest}

The authors declare no conflicts of interest regarding the publication of this paper.

\section{References}

[1] Luzi, F., Dominici, F., Armentano, I., Fortunati, E., Burgos, N., Fiori, S. and Torre, L. (2019) Combined Effect of Cellulose Nanocrystals, Carvacrol and Oligomeric Lactic Acid in PLA_PHB Polymeric Films. Carbohydrate Polymers, 223, Article ID: 115131. https://doi.org/10.1016/j.carbpol.2019.115131

[2] Kalita, N.K., Nagar, M.K., Mudenur, C., Kalamdhad, A. and Katiyar, V. (2019) Biodegradation of Modified Poly(Lactic Acid) Based Biocomposite Films under Thermophilic Composting Conditions. Polymer Testing, 76, 522-536.

https://doi.org/10.1016/j.polymertesting.2019.02.021

[3] Shojaeiarani, J., Bajwa, D.S., Stark, N.M. and Bajwa, S.G. (2019) Rheological Properties of Cellulose Nanocrystals Engineered Polylactic Acid Nanocomposites. Composites Part B: Engineering, 161, 483-489.

https://doi.org/10.1016/j.compositesb.2018.12.128

[4] Mathew, P., Oksman, K. and Sain, M. (2006) The Effect of Morphology and Chemical Characteristics of Cellulose Reinforcements on the Crystallinity of Polylactic Acid. Journal of Applied Polymer Science, 101, 300-310. https://doi.org/10.1002/app.23346

[5] Lizundia, E., Vilas, J. and León, L. (2015) Crystallization, Structural Relaxation and Thermal Degradation in Poly(L-Lactide)/Cellulose Nanocrystal Renewable Nanocomposites. Carbohydrate Polymers, 123, 256-265.

https://doi.org/10.1016/j.carbpol.2015.01.054

[6] Lizundia, E., Fortunati, E., Dominici, F., Vilas, J.L., León, L.M., Armentano, I. and Kenny, J.M. (2016) PLLA-Grafted Cellulose Nanocrystals: Role of the CNC Content and Grafting on the PLA Bionanocomposite Film Properties. Carbohydrate Polymers, 142, 105-113. https://doi.org/10.1016/j.carbpol.2016.01.041

[7] Wu, H., Nagarajan, S., Zhou, L., Duan, Y. and Zhang, J. (2016) Synthesis and Characterization of Cellulose Nanocrystal-Graft-Poly(D-Lactide) and Its Nanocomposite with Poly(L-Lactide). Polymer, 103, 365-375.

https://doi.org/10.1016/j.polymer.2016.09.070

[8] Miao, C. and Hamad, W.Y. (2016) In-Situ Polymerized Cellulose Nanocrystals (CNC)-Poly(L-Lactide)(PLLA) Nanomaterials and Applications in Nanocomposite Processing. Carbohydrate Polymers, 153, 549-558. https://doi.org/10.1016/j.carbpol.2016.08.012

[9] De Paula, E., Roig, F., Mas, A., Habas, J., Mano, V., Pereira, F. and Robin, J. (2016) Effect of Surface-Grafted Cellulose Nanocrystals on the Thermal and Mechanical Properties of PLLA Based Nanocomposites. European Polymer Journal, 84, 173-187. https://doi.org/10.1016/j.eurpolymj.2016.09.019

[10] Ioelovich, M. (2012) Optimal Conditions for Isolation of Nanocrystalline Cellulose Particles. Nanoscience and Nanotechnology, 2, 9-13. https://doi.org/10.5923/j.nn.20120202.03

[11] Graupner, N., Herrmann, A. and Müssig, J. (2009) Natural and Man-Made Cellulose Fibre-Reinforced Poly (Lactic Acid) (PLA) Composites: An Overview about 
Mechanical Characteristics and Application Areas. Composites Part A: Applied Science and Manufacturing, 40, 810-821.

https://doi.org/10.1016/j.compositesa.2009.04.003

[12] Frone, A., Berlioz, S., Chailan, J. and Panaitescu, D. (2013) Morphology and Thermal Properties of PLA-Cellulose Nanofibers Composites. Carbohydrate Polymers, 91, 377-384. https://doi.org/10.1016/j.carbpol.2012.08.054

[13] Chen, X., Kalish, J. and Hsu, L. (2011) Structure Evolution of $\alpha$-Phase Poly (Lactic Acid). Journal of Polymer Science Part B: Polymer Physics, 49, 1446-1454. https://doi.org/10.1002/polb.22327

[14] Leszczyńska, A., Radzik, P., Haraźna, K. and Pielichowski, K. (2018) Thermal Stability of Cellulose Nanocrystals Prepared by Succinic Anhydride Assisted Hydrolysis. Thermochimica Acta, 663, 145-156. https://doi.org/10.1016/j.tca.2018.03.015

[15] Cetin, N., Tingaut, P., Özmen, N., Henry, N., Harper, D., Dadmun, M. and Sebe, G. (2009) Acetylation of Cellulose Nanowhiskers with Vinyl Acetate under Moderate Conditions. Macromolecular Bioscience, 9, 997-1003.

https://doi.org/10.1002/mabi.200900073

[16] Tian, C., Yi, J., Wu, Y., Wu, Q., Qing, Y. and Wang, L. (2016) Preparation of Highly Charged Cellulose Nanofibrils Using High-Pressure Homogenization Coupled with Strong Acid Hydrolysis Pretreatments. Carbohydrate Polymers, 136, 485-492. https://doi.org/10.1016/j.carbpol.2015.09.055

[17] Fragal, E.H., Cellet, T.S., Fragal, V.H., Companhoni, M.V., Ueda-Nakamura, T., Muniz, E.C. and Rubira, A.F. (2016) Hybrid Materials for Bone Tissue Engineering from Biomimetic Growth of Hydroxiapatite on Cellulose Nanowhiskers. Carbohydrate Polymers, 152, 734-746. https://doi.org/10.1016/j.carbpol.2016.07.063

[18] Spinella, S., Re, G.L., Liu, B., Dorgan, J., Habibi, Y., Leclere, P. and Gross, R.A. (2015) Polylactide/Cellulose Nanocrystal Nanocomposites: Efficient Routes for $\mathrm{Na}$ nofiber Modification and Effects of Nanofiber Chemistry on PLA Reinforcement. Polymer, 65, 9-17. https://doi.org/10.1016/j.polymer.2015.02.048 